\title{
Eine „populistische Lektion"1: Emotionssensible Perspektiven für die politische Bildung
}

\section{Zusammenfassung}

Der vorliegende Beitrag beschäftigt sich mit der Instrumentalisierung von Emotionen als Strategie rechtspopulistischer Politik und zieht daraus Schlüsse für die politische Bildung. Die negative Bewertung von Emotionen oder deren Verdrängung aus dem politischen Diskurs bilden eine Leerstelle im Politischen. Auf Basis der Theorie der Intergruppen-Emotionen zeigt der Beitrag die Funktionen von Emotionen für Identitätsstiftung und Gruppenbildung und die Risiken für eine pluralistische Gesellschaft auf. Der Beitrag plädiert für eine stärkere Thematisierung und kritische Reflexion von Emotionen in der politischen Bildung und stellt Perspektiven für eine emotionssensible politische Bildung dar.

Schlüsselworte: politische Bildung, Rechtspopulismus, Emotionen, Intergruppen-Emotionen, Identität

\begin{abstract}
This article addresses the instrumentalization of emotions as a strategy of right-wing populist politics and draws conclusions concerning civic education. The negative evaluation of emotions or their suppression from the political discourse forms a void in the political discourse. Based on the intergroup-emotions-theory the article shows the functions of emotions for identity building and group formation and the risks for a pluralistic society. The article argues for a stronger thematization and critical reflection of emotions in civic education and outlines perspectives for an emotion-sensitive civic education.
\end{abstract}

Keywords: civic education, right-wing populism, emotions, intergroup-emotions, identity

\section{Rechtspopulismus als Herausforderung}

Ist eine Auseinandersetzung mit Rechtspopulismus oder mit einem der von Rechtspopulist/inn/en häufig vorgebrachten Themen Gegenstand der Debatte, gehen die Wogen hoch: Positionierungen sind von Gefühlsäußerungen wie Wut, Ablehnung,
Aggression oder auch Begeisterung begleitet. Stellungnahmen werden mit Vorurteilen und Ressentiments verknüpft und sind emotional aufgeladen. Oft wird in diesem Zusammenhang beklagt, dass eine sachliche Diskussion nur schwer oder kaum mehr möglich sei und es wird vor zunehmender Emotionalisierung politischer Diskurse gewarnt. An die Stelle von Fakten, Tatsachen und rationalen Argumenten seien Fake News, gefühlte Wahrheiten und subjektive Empfindungen getreten.

Tatsächlich gehört das Ansprechen und Instrumentalisieren von Emotionen zum Kernrepertoire rechtspopulistischer Politik. Dies wird häufig mit Strategien der Personalisierung, Simplifizierung, Moralisierung, Dramatisierung oder Polarisierung verbunden. „Wut, Verachtung, Abwertung“ (Zick \& Küpper, 2015, S. 11) sind der zentrale „Dreiklang“ des Rechtspopulismus, der auch als Schirmbegriff verstanden werden kann, unter dem sich „Wut und gefährlicher Hass zu einer nur noch schwer zu differenzierenden Gemengelage vermischt haben“ (Quent, 2017, S. 58). Dadurch wird einerseits bei der Auseinandersetzung mit Rechtspopulismus vermehrt die Bedeutung von Emotionen in politischen Prozessen in den Blick gerückt. Zögerlich finden so auch Emotionstheorien und Erkenntnisse aus der Emotionsforschung Einzug in die Politikwissenschaft. Andererseits zeigt sich eine vorwiegend negative Bewertung von Gefühlen ${ }^{2}$ im politischen Rahmen: Sie gelten als irrational und werden daher als Störungen der politischen Ordnung gesehen, die es zu vermeiden gilt (Besand, 2015; Heidenreich, 2012).

Diesem Gedanken folgend, behandelt auch die politische Bildung Emotionen entweder kaum oder versucht, diese im Sinne des Rationalitätsideals zu überwinden. Erfahrungen aus der Unterrichtspraxis zeigen gleichwohl, dass Schüler/-innen besonders anfällig für die emotionale Komponente rechtspopulistischer Bedrohungsszenarien sind (Elverich, 2017, S. 58). Um solchen emotionalen Manipulationsversuchen und der damit verknüpften gesellschaftlichen Polarisierung und Re-Nationalisierung entgegenzuwirken, wird vielfach die Forderung nach mehr Aufklärung laut; durch eine Fokussierung auf Fakten sollen Emotionen so quasi beseitigt werden. Dabei wird übersehen, dass sich Emotionen weder aus Prozessen der Politik noch aus jenen der Bildung 
gänzlich verbannen lassen: Emotionen sind ein grundlegender und integrativer Teil dessen, wie wir uns selbst, unsere Mitmenschen und die Welt insgesamt wahrnehmen, interpretieren sowie in Bezug zueinander setzen und zu welchen Urteilen und Handlungsentscheidungen wir gelangen (Weber, 2016; Weber-Stein, 2017). Dabei können Emotionen sowohl destruktiv als auch konstruktiv, demokratiegefährdend oder demokratieförderlich, spaltend oder verbindend, ermutigend oder hemmend wirken. Dies ist abhängig davon, wie mit ihnen umgegangen wird (Charim, 2017, S. 10; Kleres \& Albrecht, 2015, S. 3ff. u. 13). Daher käme auch der politischen Bildung die wichtige, aber bisher kaum wahrgenommene Aufgabe zu, die Rolle von Emotionen anzuerkennen und konstruktive Umgangsmöglichkeiten zu entwickeln. Im Folgenden möchte ich umreißen, wie sich die politische Bildung dieser Aufgabe in Zeiten rechtspopulistischer Konjunktur annähern kann.

\section{Rechtspopulismus: Politik der Gefühle?}

Um zu analysieren, wie Rechtspopulismus Emotionen anspricht und Identifikation stiftet, ist die breit rezipierte Definition des niederländischen Politikwissenschaftlers Cas Mudde hilfreich. Er versteht Populismus im Allgemeinen „as an ideology that considers society to be ultimately separated into two homogeneous and antagonistic groups, ,the pure people versus ,the corrupt elite', and which argues that politics should be an expression of the volonté générale (general will) of the people" (Mudde, 2004, S. 543). In dieser Definition stecken die drei Kernmerkmale des Populismus: Erstens die Bezugnahme auf „das Volk“, das sowohl Adressat von als auch Legitimationsgrundlage für populistische Politik ist; zweitens die Moralisierung von Politik (das „gute“ Volk gegen die „böse“ Elite); und drittens die antagonistische Polarisierung dieser Gruppen (dazu: Priester, 2017, S. 9; Wodak, 2016, S. 25).

Dieses dünne ideologische Substrat bietet Anknüpfungspunkte für unterschiedliche politische Programme und ist $\mathrm{zu}$ nächst weder dem rechten noch dem linken politischen Spektrum eindeutig zuzuordnen (Decker, 2017, S. 22). Wie das Volk und seine antagonistischen Gegner genau definiert und in welchem konkreten Verhältnis diese konstruiert werden, schlägt eine Brücke zur inhaltlichen Bestimmung populistischer Parteien. Die Kritik an der Elite und dem Establishment eint sowohl rechts- als auch linkspopulistische Ansätze. Dabei ist Linkspopulismus stärker antikapitalistisch ausgerichtet und betont aufseiten des Volkes Vielfalt und Solidarität. Demgegenüber definiert Rechtspopulismus das Volk vor allem als ethnisch und kulturell homogen (Wodak, 2016, S. 64; Decker 2017, S. 22f.). Im rechtspopulistischen Narrativ wird das „brave“, „fleißige“ und "gute“ Volk daher nicht nur von „denen da oben“ (der „korrupten, ausbeuterischen Elite“) gefährdet, sondern auch von „denen da draußen“ (den „Auslände$\mathrm{r} /$ inne/n“ und Migrant/inn/en), von „denen da unten“ (den „Sozialschmarotzern“ und „Leistungsverweigerern“) und schlussendlich auch von jenen Menschen, die eine andere politische Meinung vertreten und die mitunter als „Gutmenschen“"oder gar „Volksverräter" abgekanzelt werden (Pelinka, 2002, S. 284f.; Wodak, 2016, S. 18 u. 27). Charakteristisch ist daher neben einer Anti-Establishment-Haltung und Fremdenfeindlichkeit auch eine anti-pluralistische Haltung, die von Kritik bis zur Ablehnung konsensualer Demokratie reichen kann.

Um zu analysieren, wie solch identitätsrelevante $\mathrm{Zu}$ schreibungen in emotionaler Hinsicht wirken, stütze ich mich im Folgenden auf die Theorie der Intergruppen-Emotionen, die ihrerseits an die Social Identity Theory von Henri Tajfel (1979; 1982) anknüpft. Diese geht davon aus, dass es Emotionen gibt, die durch eine solche soziale Kategorisierung ausgelöst werden und die soziale, aber auch politische Beziehungen zwischen Gruppen regeln (Seger, Smith \& Mackie, 2009, S. 460f.). Gruppenzugehörigkeiten haben demzufolge Einfluss auf das emotionale Erleben von Menschen sowie auf deren Einstellungen und Verhaltensweisen: Wird die Zugehörigkeit zu einem Kollektiv durch bestimmte Symbole, Begriffe, Rituale, physische Nähe zu anderen Gruppenmitgliedern oder auch durch Diskriminierungserfahrungen aktiviert, treten die individuellen Belange und Interessen hinter jene der Gruppe zurück. Somit verändern sich auch die Parameter, anhand derer Menschen eine Situation, ein Ereignis oder Personen und Gruppen in emotionaler Hinsicht bewerten. Dadurch ist es möglich, dass man sich beispielsweise als Individuum sehr glücklich oder optimistisch fühlen kann, aus Sicht einer bestimmten Gruppe jedoch nicht (Mackie, Smith \& Gay, 2008, S. 1869). Diese Theorie ist insbesondere in solchen Kontexten relevant, in denen es um tatsächliche, vorgestellte oder befürchtete Konflikte, um Konkurrenz, kollektive Identität(en) und das kulturelle Selbstverständnis geht (Salmela \& Scheve, 2017, S. 15). Sie bietet sich daher als Raster für eine Analyse rechtspopulistischer Strategien an.

Rechtspopulismus zieht klare Demarkationslinien zwischen der Ingroup (dem „Volk“) und der Outgroup (den „Anderen“); diese Linien schaffen ein starkes Zusammengehörigkeitsgefühl, gerade weil damit eine Spaltung zu einer anderen Gruppe einhergeht. In Form einer Stereotypisierung werden der Ingroup dabei primär positive, der Outgroup hingegen primär negative Eigenschaften, Einstellungen, Haltungen und Verhaltenstendenzen zugeschrieben. Das Selbstbewusstsein der eigenen Gruppe und der Zusammenhalt werden gestärkt, gleichzeitig bieten diese Konstruktionen aber auch einen idealen Nährboden für vereinfachende Generalisierungen, Feindbilder und Sündenbockrhetorik (Ötsch \& Horaczek, 2017, S. 72ff.). Dabei wird einerseits an vorhandene kulturelle, ethnische oder nationale Identität(en) angeknüpft, andererseits werden diese aber auch diskursiv erzeugt (Salmela \& Scheve, 2017, S. 16).

Diese Zuschreibungen stehen den Mitgliedern als stereotypes Wissen zur Verfügung, das wiederum beeinflusst, welche Emotionen zutage treten - die Gruppenmitglieder reagieren so, wie sie glauben, dass sie "typischerweise“ reagieren sollen. Rechtspopulist/inn/en schaffen es dabei, eben dieses stereotype Wissen um affektive Reaktionen für die Gruppe bereitzustellen und durch langfristige Wiederholung als Realität festzulegen. So etablieren sie beispielsweise, dass das "Volk“ sich etwa von den religiösen Praktiken von Muslim/inn/en verletzt oder bedroht oder es sich durch antidiskriminierende Gleichstellungsmaßnahmen benachteiligt fühle. Die Etablierung sozialer Normen durch Gruppen führt auch zur Festlegung emotionaler Reaktionen gegenüber bestimmten Themen, Ereignissen oder Gruppen. Dies führt zu einer zunehmenden Konvergenz der Gefühlslagen sowie zu einer Gleichschaltung von Interpretationsmustern und normativen Erwartungen innerhalb der Gruppe. Diese haben wiederum Einfluss auf das Verhalten (Salmela \& Scheve, 2017, S. 16). Je höher der Identifikationsgrad mit einer bestimmten Gruppe, desto wahrscheinlicher ist es, dass die Individualität einer Person zugunsten von durch Selbststereotypisie- 
rung festgelegter Gruppeneigenschaften, -einstellungen und -verhaltensweisen zurücktritt (Mackie, Smith \& Gay, 2008, 1867ff.; Seger, Smith \& Mackie, 2009, 460ff.). Andere soziale Einflüsse verlieren in weiterer Folge an Bedeutung. Die Gruppe wird zu einem zentralen, mitunter sogar dominierenden Teil der eigenen Identität.

Wut und Angst sind die zwei Hauptemotionen, die Rechtspopulist/inn/en durch die von ihnen entworfenen personalisierten Bedrohungsszenarien ansprechen. Dabei führt Wut als Intergruppen-Emotion zu dem Wunsch, die Outgroup zu konfrontieren, zu verletzen oder gar zu attackieren. Wut kann sich dabei in unterschiedlichen Formen äußern: Beschimpfungen, Beschuldigungen, Drohungen, fehlende Empathie gegenüber Anderen, Exklusion, Diskriminierung und die Anwendung physischer Gewalt (Mackie, Smith \& Gay, 2008, S. 1874f.; vgl. dazu Ötsch \& Horaczek, 2017, S. 63). Der Wut ist in diesem Zusammenhang eine Eskalationsdynamik eingebettet. Denn Populismus hat durch Tabubrüche, Provokationen und der scharfen Kritik an herrschenden Verhältnissen den Impuls, sich fortlaufend selbst zu radikalisieren:

"Tatsächlich hat die ausgrenzende oder beleidigende Sprache gegenüber den Feindgruppen der Rechtspopulisten seit einigen Jahren an Aggressivität zugenommen. Hassrede wird durch digitale Medien beflügelt, in denen auch Einzelne oder relativ kleine ideologische Gruppen eine enorme Breitenwirkung entfalten können. Die drastisch verschärfte Sprache dürfte nicht zuletzt dazu beigetragen haben, dass in einigen Ländern (z.B. Ungarn, Deutschland) die Hemmschwelle zu physischer Gewalt gesunken und die Zahl rassistischer Angriffe drastisch gestiegen ist" (Decker \& Lewandowsky, 2017, S. 19).

Eine Verrohung der Sprache gegenüber einer bestimmten Gruppe führt in der Regel also auch zu einem härteren Umgang mit dieser Gruppe. Die Intergruppen-Emotion Angst führt hingegen zu einer Distanzierung zur Outgroup. Es ist dabei egal, ob die Ursachen für das Gefühl real oder imaginiert sind, sich die Angst auf ein Ereignis in der Zukunft, der Gegenwart oder gar der Vergangenheit bezieht. Das Gefühl ist in seinen Auswirkungen real (Mackie, Smith \& Gay, 2008, S. 1874f.).

Weder Wut und Aggression noch Angst und Distanz sind für das Zusammenleben in heterogenen Gesellschaften förderlich. Sie führen zu Polarisierung, sozialen Gräben, einem Bedürfnis nach weiterer Homogenisierung und destruktiven Konfrontationen. Sie führen aber auch dazu, dass der innere Zusammenhalt der Ingroup gestärkt wird. Je stärker und öfter die antagonistische Spaltung der Gesellschaft und die damit einhergehenden Bedrohungsszenarien beschworen werden, desto wirkmächtiger werden diese Differenz- und Identitätskonstruktionen. In ihrer radikalsten Zuspitzung wird der Outgroup der Status vollwertiger Menschen abgesprochen und sie wird aus dem Kreis derjenigen ausgeschlossen, für die rechtliche und/ oder moralische Grundsätze Geltung haben. Wenn die Moral und Tugend exklusiv für die Ingroup beansprucht werden, so haben die amoralischen "Anderen" es auch nicht verdient, Rechte zu haben. Dies ist ein Argument, das einen graduellen Prozess der Entrechtung bis hin zum Absprechen der Menschenrechte nach sich ziehen kann (Scherr, 2011, S. 81; Ötsch \& Horaczek, 2017, S. 31; vgl. auch Häusler \& Roeser, 2014, S. 13).

\section{Leerstelle Emotion: \\ Die „populistische Lektion“}

Was können wir aus der Analyse rechtspopulistischer Emotionalisierungsstrategien lernen? Zunächst sicherlich, dass Gefühle das Politische stärker als bisher gedacht mitformen und gestalten. Die angenommene Dichotomie zwischen Emotionalität und Rationalität, welche Politik und Wissenschaft geprägt und zur Exklusion von Gefühlen geführt hat, ist angesichts neuer Erkenntnisse aus der Emotionsforschung nicht länger haltbar. Emotion und Ratio sind auf komplexe Art und Weise miteinander verbunden (Heidenreich, 2012, S. 9; Petri, 2018, S. 44). In Emotionen spiegeln sich Wertvorstellungen, Einstellungen, Haltungen, Überzeugungen und Bedürfnisse einer Person oder Gruppe. Gefühle verfügen daher auch über eine kognitive Komponente und sind an allen Phasen der Informationsverarbeitung beteiligt. Dadurch helfen sie uns bei der Organisation und Kategorisierung unserer Lebenserfahrungen. Emotionen stiften somit Orientierung und bestimmen maßgeblich mit, wie wir uns selbst und die Welt sehen und in Bezug zueinander setzen. Sie regeln Zugehörigkeiten sowie die sozialen und politischen Relationen zwischen Gruppen und sind eng mit Identitätsfragen verknüpft. Sie werden durch soziale und politische Faktoren beeinflusst, greifen aber auch ihrerseits in die gesellschaftliche und politische Ordnung ein. Gefühle werden daher nicht passiv erlitten, sondern sind modulier- und regulierbar. Dementsprechend müssen sie sich - im Unterschied zu impulsiven Affekten - auch nicht entladen, sondern können unterdrückt oder verändert werden (Nussbaum, 2001; Weber, 2016; Weber-Stein, 2017; Scherke, 2009).

Eine funktionierende Demokratie muss daher Gefühle im Diskurs integrieren: Wenn alle politischen Subjekte Gefühle haben, sind Emotionen auch politisch relevant. Sie schwingen bei jeder politischen Auseinandersetzung mit. Werden Emotionen tabuisiert, verschwinden sie nicht einfach, sondern bewegen sich als „vagabundierendes Potential“ (Charim, 2017, S. 10). Sie können sowohl in eine produktive als auch in eine destruktive Richtung wirken. Im politischen Sinn gibt es keine „guten“ oder „schlechten“, „demokratischen“ oder „antidemokratischen "Emotionen. Wie sie wirken, ist davon abhängig, wie mit ihnen umgegangen wird.

Darin sieht Charim „die populistische Lektion“: Rechtspopulistische Akteurinnen und Akteure haben erkannt, dass Gefühle eine Leerstelle im Politischen bilden und haben diese besetzt. In Zeiten, in denen Identitätsbelange zunehmend klassische Interessenskonflikte überlagern, setzen Rechtspopulist/inn/en ein klares Identitätsangebot. Dieses funktioniert vornehmlich durch die feindbildgestützte identitäre Schließung von „Wir“ gegen „die Anderen“, was in der Abwertung, Diskriminierung oder Gewaltanwendung gegenüber der Outgroup münden kann. Gleichzeitig werden aber auch positive Gefühle gegenüber der Wir-Gruppe erzeugt oder verstärkt, die in einer starken Bindung an eine Partei und einer Zunahme an politischem Interesse und Engagement münden. Als Antwort auf die rechtspopulistische Konjunktur bräuchte es eine Politik, die „Emotionen berührt“ und ein „Identitätsangebot macht“ (Charim, 2017, S. 12). Gefordert ist eine Politik, die Schwarz-WeißDenken überwindet und sich jenseits der Abwertung anderer verortet. 


\section{Emotionssensible Perspektiven für die politische Bildung}

Damit werden wesentliche Impulse für den Umgang mit Rechtspopulismus im Rahmen der politischen Bildung benannt: Emotionen, Pluralität und demokratische Konfliktfähigkeit. Der Erfolg der rechtspopulistischer Emotionalisierung zeigt, dass Gefühle und Identitätsbelange nicht auszuklammern, sondern als wichtige Faktoren des Politischen anzuerkennen sind. Nur so können Reflexionsprozesse angeregt werden, die auch vor emotionaler Manipulation schützen können. Dazu muss die politische Bildung aber auch Konzepte für einen konstruktiven Umgang mit Gefühlen entwickeln, die jenseits von Homogenisierungsbestrebungen, Polarisierung und Feindbildkonstruktionen stehen. Stattdessen gilt es, ihr Potential für eine lebendige und pluralistische Demokratie mit starkem zivilgesellschaftlichem Engagement auszuloten und in Bezug zu demokratischen Werten wie Gleichheit, Solidarität, Toleranz und Gerechtigkeit zu setzen.

Das Prinzip der Kontroversität als Leitlinie politischer Bildungsarbeit trägt in diesem Sinne wesentlich zur Ausbildung von Mündigkeit bei. Es hilft beim Umgang mit Rechtspopulismus, der antipluralistischen Tendenz durch das Aufzeigen von Alternativen entgegenzuwirken. Bereits Hannah Arendt hat darauf hingewiesen, dass sich das Individuum seine Meinung nicht alleine bildet und anschließend das propagiert, was es sich im „Kopf bereits zurechtgelegt habe“ (Arendt, 2012, S. 63). Stattdessen braucht es diskursive Auseinandersetzung mit anderen Standpunkten, Haltungen und Sichtweisen im Sinne „bezogener Urteilsbildung“ (Schröder, 2016, S. 305). Kontroversität kann hier für Irritationen sorgen, die geschlossene Weltbilder verhindern und vorgefestigte Meinungen infrage stellen. Dieser Effekt tritt allerdings nicht ein, wenn es beim bloßen Präsentieren von unterschiedlichen Positionen bleibt. Um eine tiefgreifende Auseinandersetzung jenseits von belanglosem „Entweder/ Oder" (Hammermeister, 2016, S. 174) oder der Aneinanderreihung von „beliebigen Meinungsgirlanden“" (Weißeno, 1996, S. 110) anzuregen, muss um diese Positionen gestritten und gerungen werden. Das gehört zur Ausbildung einer Streit- und Konfliktkultur, wie sie für Demokratien wesentlich ist. Dabei werden Werte, Urteile, Haltungen und Meinungen infrage gestellt - innere Orientierungspunkte also, die emotional behaftet und verankert sind. Geraten diese ins Wanken, kann es folglich auch zu starken emotionalen Reaktionen kommen. Das birgt sowohl Gefahr als auch Potential in sich, denn einerseits können so die „Tiefenschichten“ der politischen Urteilsbildung erreicht werden, andererseits besteht auch die Gefahr der Polarisierung und Ablehnung.

Wird eine Person mehreren, in Widerspruch zueinander stehenden Wahrnehmungen, Gedanken, Meinungen, Einstellungen, Wünschen oder Absichten ausgesetzt, entsteht kognitive Dissonanz (Festinger, 1962). Diese wird als unangenehm empfunden, weil sie im Individuum starken Druck erzeugt und im Bestreben resultiert, Bestätigung statt Unstimmigkeiten zu erfahren. Eine Revision eigener Meinungen findet selten statt, ist aber nicht ausgeschlossen. Eine förderliche Bedingung dafür ist, das sich in Dissonanz befindliche Gegenüber nicht durch Moralisierungen, Vorwürfe oder Ähnliches in die Ecke zu drängen. Denn dann würde die betreffende Person vehement versuchen, ihre Meinung zu verteidigen und ihre Position weiter zu verfestigen. Das Resultat ist Abwehr, sodass kein konstruktiver Dialog mehr möglich ist. Wenn sich aber das Gegenüber in seiner Identität und Gruppenzugehörigkeit hingegen positiv wahrgenommen fühlt, wird es offen für Dialog und Widerspruch. Spalten rechtspopulistische Emotionalisierungsstrategien Gruppen, so kann ein Berücksichtigen von Emotionen (wieder) Diskursräume eröffnen, welche die Ablehnung bestimmter inhaltlicher Positionen bei gleichzeitiger Wahrung der Beziehungsebene begünstigt.

Aufgrund ihrer motivationalen Kraft sind Emotionen aber nicht nur an der Bildung, Reflexion und Änderung von Urteilen beteiligt, sondern auch maßgeblich daran, welche Handlungen gesetzt werden. Normen und Werte alleine sind nicht ausreichend, um sich in Politik und Gesellschaft einzubringen. Es braucht auch Courage, die Zuversicht, Dinge ändern zu können, aber auch Empörung über wahrgenommene Ungerechtigkeiten oder Empathie für jene, deren Rechte verletzt werden (Breit, 2016, S. 23). Ohne diese Aspekte wären etwa keine sozialen Bewegungen denkbar, die ein hohes Maß an langandauerndem Engagement erfordern. Die mit den eigenen Vorstellungen von einem gelingenden Leben verknüpften Normen und Werte sind dabei in besonderer Weise emotionsbehaftet und motivational (Nussbaum, 2001, S. 211). Diese Vorstellungen sollen aber nicht nur auf das Selbst und das Durchsetzen seiner Interessen abzielen, sondern auch die Perspektive des kollektiven Handelns in Betracht ziehen, die „über eigene Interessen hinaus Solidarität an den Tag" (Schiele, 1996, S. 7) legt. Die demokratische Verantwortung besteht auch darin, die Autonomie und das Wohl des Einzelnen in Zusammenhang mit der Autonomie und dem Wohl Anderer zu sehen. Grundlegende Voraussetzung dafür ist die Fähigkeit, sich in die Interessen, Denkweisen und Befindlichkeiten von anderen hineinzuversetzen und den Anderen als eigenständige Person mit eigenen Rechten anzuerkennen.

Solidarität ist ohne Empathie und Zugehörigkeit nicht denkbar. In einer zunehmend globalisierten Welt, in der sie auch über nationale Grenzen hinweg Geltung haben soll, stellt dies Menschen vor erhebliche Herausforderungen. Rechtspopulismus stiftet über Feindbilder Zusammengehörigkeit: Diese Strategie ist deshalb so erfolgreich, weil es sich bei dem „Volk“ um eine imaginäre Gruppe ohne konkreten Erfahrungs- und Kommunikationszusammenhang handelt. Als solche steht die Gruppe in besonderem Maße vor der Herausforderung, Zugehörigkeit zu ermöglichen (Scherr, 2011, S. 81). Ein gemeinsames, feindliches Gegenüber stiftet hier Zusammenhalt und Identifikation. Angesichts dessen ist es fraglich, ob die Ausbildung einer Identität als Weltbürgerin und Weltbürger, die Diversität und Differenz beinhaltet, möglich ist. Eine Fokussierung auf alternierende Gruppenzugehörigkeiten kann angesichts der Erkenntnisse aus der Intergruppen-Theorie aber starre Polarisierungen aufweichen und zur Förderung von Austausch und Dialog beitragen.

\section{Fazit}

Anstelle des Rekurses auf ein vor allem ethnisch-kulturell homogenes Nationalvolk braucht es Konzepte, die Orientierung und Handlungsperspektiven in einer heterogenen und globalisierten Gesellschaft stiften. Anstelle einer Reduktion von Komplexität zugunsten einfacher, dichotomer Schemata muss der 
Umgang mit Ambivalenzen, Ambiguitäten, Unsicherheiten und Kontroversen geschult werden. Diese Ideen sind nicht grundlegend neu, wurden bisher allerdings zu wenig im Hinblick auf die Rolle und Funktion von Emotionen reflektiert. Gerade angesichts des Rechtspopulismus mit seiner antipluralistischen Spaltungstendenz und Eskalationsdynamik braucht es Anregungen aufrichtigen Dialogs. Zudem braucht es eine Fokussierung auf die verbindende Wirkung von Emotionen und ihre Rolle zur Bearbeitung kontroverser Auseinandersetzungen mit politischen Themen. Eine kritische Reflexion von Emotionen ist dabei grundlegende Voraussetzung: Sie kann einen Aspekt demokratischer Willensbildung darstellen, bei der Emotionen weder als unnötig, infantil oder per se gefährlich abgetan werden noch als diffuse Basis für politische Einstellungen, Urteile und Handlungen unhinterfragt bleiben. Vielmehr ist zu unterstreichen, dass Emotionen zu chancenreichen Dialogprozessen einer lebendigen politischen Auseinandersetzung beitragen, die konstruktiven Dissens pflegt. Ein emotionssensibler Ansatz politischer Bildung steht nicht im Widerspruch zu den Prinzipien von Mündigkeit, Emanzipation, Solidarität und Konfliktfähigkeit, sondern trägt zur erfolgreichen Ausbildung dieser Bildungsziele bei.

\section{Anmerkungen}

1 In Anlehnung an Isolde Charim (2017).

2 Als Gefühl wird vor allem im deutschsprachigen Raum das subjektive Erleben einer Emotion beschrieben (Brandstätter et al., 2013, S. 130;). Diese Unterscheidung ist jedoch gerade ausgehend von einem kognitivistischen Emotionsbegriff, der diesem Artikel zugrunde liegt, schwierig zu treffen. Im Folgenden werden Gefühle und Emotionen als Synonyme verwendet.

\section{Literatur}

Arendt, H. (2012). Das Urteilen. Texte zu Kants Politischer Philosophie. Dritter Teil „Vom Leben des Geistes". München \& Zürich: Piper.

Besand, A. (2015). Gefühle über Gefühle. Zum Verhältnis von Emotionalität und Rationalität in der politischen Bildung. In K.-R. Korte (Hrsg.), Emotionen und Politik. Begründungen, Konzeptionen und Praxisfelder einer politikwissenschaftliche Emotionsforschung.(S.213-224).Baden-Baden:Nomos.doi.org/10.5771/9783845263380213

Brandsätter, V., Schüler, J., Puca, R. M. \& Lozo, L. (2013). Motivation und Emotion. Angewandte Psychologie für Bachelor. Berlin: Springer. doi.org/10.1007/978-3-64230150-6

Breit, G. (2016). Mit Leidenschaft und Augenmaß zugleich. Zum Spannungsverhältnis von Rationalität und Emotionalität im Politikunterricht. Schwalbach/Ts.: Wochenschau.

Charim, I. (2017). Das pluralisierte Individuum. Analysen \& Impulse, kommentpapers 3, S. 6-13.

Decker, F. (2017). Populismus in Westeuropa. Theoretische Einordnung und vergleichende Perspektiven. In G. Diendorfer, G. Sandner \& E. Turek (Hrsg.), Populismus - Gleichheit - Differenz. Herausforderungen für die Politische Bildung. (S. 11-28). Schwalbach/Ts.: Wochenschau.

Decker, F. \& Lewandowsky, M. (2017). Rechtspopulismus. Erscheinungsformen, Ursachen und Gegenstrategien. In Bundeszentrale für Politische Bildung (Hrsg.), Dossier Rechtspopulismus. (S. 22-34), Zugriff am 29.01.2019, www.bpb.de/system/ files/pdf_pdflib/pdflib-241384.pdf.

Elverich, G. (2017). Zeitgemäße Ansätze politischer Bildung in der Schule angesichts rechtspopulistischer Tendenzen. In B. Jungkamp \& M. John-Ohnesorg (Hrsg.), Politische Bildung in der Schule. Zeitgemäße Ansätze in Zeiten des Populismus (Schriftenreihe des Netzwerk Bildung; S. 57-62). Berlin: Friedrich-Ebert-Stiftung.

Festinger, L. (1962). A theory of cognitive dissonance, Stanford: Stanford University Press.

Hammermeister, J. (2016). Macht- und Herrschaftsverhältnisse. Ein blinder Fleck des Beutelsbacher Konsens. In B. Widmaier \& P. Zorn (Hrsg.), Brauchen wir den Beutelsbacher Konsens? Eine Debatte der politischen Bildung. (S. 171-178). Bonn: Bundeszentrale für politische Bildung.

Häusler, A. \& Roeser, R. (2014). Rechtspopulismus in Europa und die rechtspopulistische Lücke in Deutschland. Erfurt: Mobit.
Heidenreich, F. (2012). Versuch eines Überblicks. Politische Theorie und Emotionen. In F. Heidenreich \& G. Schaal (Hrsg.), Politische Theorie und Emotionen. (S. 9-26). Baden-Baden: Nomos. doi.org/10.5771/9783845238364-9

Kleres, J. \& Albrecht, Y. (2015). Die verbindende und ambivalente Sozialität der Gefühle. In J. Kleres \& Y. Albrecht (Hrsg.), Die Ambivalenz der Gefühle. Über die verbindende und widersprüchliche Sozialität von Emotionen. (S. 1-15). Wiesbaden: Springer. doi.org/10.1007/978-3-658-01654-8

Mackie, D. M., Smith, E. R. \& Gay, D. G. (2008). Intergroup emotions and intergroup relations. Social and Personality Psychology Compass, 2(5), 1866-1880. doi. org/10.1111/j.1751-9004.2008.00130.x

Mudde, C. (2004). The Populist Zeitgeist. Government and Opposition, 39(3), 541-563. doi.org/10.1111/j.1477-7053.2004.00135.x

Nussbaum, M. (2001). Upheavals of Thought. The Intelligence of Emotions. New York: Cambridge University Press. doi.org/10.1017/CBO9780511840715

Ötsch, W. \& Horaczek, N. (2017). Populismus für Anfänger. Anleitung zur Volksverführung. Frankfurt/M.: Westend.

Pelinka, A. (2002). Die FPÖ in der vergleichenden Parteienforschung. Zur typologischen Einordnung der Freiheitlichen Partei Österreichs. Österreichische Zeitschrift für Politikwissenschaft, 31(3), 281-290.

Petri, A. (2018). Emotionssensibler Politikunterricht. Konsequenzen aus der Emotionsforschung für Theorie und Praxis politischer Bildung. Schwalbach/Ts.: Wochenschau.

Priester, K. (2017). Das Syndrom Rechtspopulismus. In Bundeszentrale für politische Bildung (Hrsg.), Dossier Rechtspopulismus. (S. 7-12), Zugriff am 28.08.2018 www. bpb.de/system/files/pdf_pdflib/pdflib-241384.pdf.

Quent, M. (2017). Rechtspopulismus und Radikalisierung. Wenn Wut zu Hass wird. Bürger im Staat, 67(1), 55-62.

Salmela, M. \& von Scheve, C. (2017). Die Emotionen des Rechtspopulismus. Latenz. Journal für Philosophie und Gesellschaft, Arbeit und Technik, Kunst und Kultur, 2, 11-37, Zugriff am: 10.01.2019 https://www.polsoz.fu-berlin.de/soziologie/arbeitsbereiche/ emotionen/team/Professur/preprints/Salmela-Scheve_EmotionenRechtspopulis mus_preprint.pdf.

Scherke, K. (2009). Emotionen als Forschungsgegenstand der deutschsprachigen Soziologie. Wiesbaden: Springer. doi.org/10.1007/978-3-531-91739-9

Scherr, A. (2011). Rassismus oder Rechtsextremismus? In C. Melter \& P. Mecheril (Hrsg.), Rassismuskritik Band 1: Rassismustheorie und-forschung. (S. 75-97). Schwalbach/Ts.: Wochenschau.

Schiele, S. (2017). Populismus, Extremismus und der Beutelsbacher Konsens. Möglichkeiten und Grenzen Politischer Bildung. In G. Diendorfer, G. Sandner \& E. Turek (Hrsg.), Populismus - Gleichheit - Differenz. Herausforderungen für die Politische Bildung. (S. 40-48). Schwalbach/Ts.: Wochenschau.

Schröder, A. (2016). Emotionale und intersubjektive Dimensionen der (jugendlichen) Urteilsbildung. Zur Kritik am „Neutralitätsgebot“ des Beutelsbacher Konsens. In B. Widmaier \& P. Zorn (Hrsg.), Brauchen wir den Beutelsbacher Konsens? Eine Debatte der politischen Bildung. (S. 303-313). Bonn: Bundeszentrale für politische Bildung.

Seger, C. R., Smith, E. R. \& Mackie, D. M. (2009). Subtle activation of a social categorization triggers group-level emotions. Journal of Experimental Psychology, 45, 460-467. doi.org/10.1016/j.jesp.2008.12.004

Tajfel, H. (1982). Social psychology of intergroup relations. Annual Review of Psychology, 33(1), 1-39. doi.org/10.1146/annurev.ps.33.020182.000245

Tajfel, H. \& Turner, J. (1979). An integrative theory of intergroup conflict. In W. G. Austin \& S. Worchel (Hrsg.), The social psychology of intergroup relations. (S. 33-57). Monterey: Brooks/Cole.

Weber, F. (2016). Emotion und Kognition in der Politischen Bildung. Überlegungen aus emotionstheoretischer Sicht. In C. Deichmann \& M. May (Hrsg.), Politikunterricht verstehen und gestalten. (S. 165-183). Wiesbaden: Springer. doi.org/10.1007/9783-658-11859-4_10

Weber-Stein, F. (2017). Die affektive Dimension des hermeneutischen Zirkels. Emotionale Bedingungsfaktoren der politischen Werturteilsbildung. zeitschrift für didaktik der gesellschaftswissenschaften, 1, 54-73.

Weißeno, G. (1996). „Was in Wissenschaft und Politik kontrovers ist, muß auch im Unterricht kontrovers dargestellt werden." Probleme bei der Umsetzung dieser Forderung. In S. Schiele \& H. Schneider (Hrsg.), Reicht der Beutelsbacher Konsens? (S. 107-127). Schwalbach/Ts.: Wochenschau.

Wodak, R. (2016). Die Politik mit der Angst. Zur Wirkung rechtspopulistischer Diskurse. Wien/Hamburg: Konturen.

Zick, A. \& Küpper, B. (2015). Wut, Verachtung, Abwertung. Rechtspopulismus in Deutschland. Bonn: J.H.W. Dietz.

\section{Kristina Langeder}

ist Referentin für politische Bildung im Friedensbüro Salzburg und angehende Lehrerin mit den Interessensschwerpunkten Extremismus, Populismus, Diversität und politischer Psychologie. Sie forscht aktuell zu Möglichkeiten einer emotionssensiblen Auseinandersetzung mit Rechtspopulismus in der politischen Bildung. 